\title{
Perspectives of Long-Haul WDM Transmission Systems Based on Phase-Insensitive Fiber-Optic Parametric Amplifiers
}

\author{
Jazayerifar, M.; Sackey, I.; Elschner, R.; Da Ros, Francesco; Richter, T.; Meuer, C.; Peucheret, \\ Christophe; Schubert, C.; Petermann, Klaus
}

\section{Published in:}

Proceedings of 2015 IEEE Photonics Society Summer Topicals Meeting Series

Link to article, DOI:

10.1109/PHOSST.2015.7248272

Publication date:

2015

Document Version

Peer reviewed version

Link back to DTU Orbit

\section{Citation $(A P A)$ :}

Jazayerifar, M., Sackey, I., Elschner, R., Da Ros, F., Richter, T., Meuer, C., Peucheret, C., Schubert, C., \& Petermann, K. (2015). Perspectives of Long-Haul WDM Transmission Systems Based on Phase-Insensitive Fiber-Optic Parametric Amplifiers. In Proceedings of 2015 IEEE Photonics Society Summer Topicals Meeting Series (pp. 211-212). IEEE. https://doi.org/10.1109/PHOSST.2015.7248272

\section{General rights}

Copyright and moral rights for the publications made accessible in the public portal are retained by the authors and/or other copyright owners and it is a condition of accessing publications that users recognise and abide by the legal requirements associated with these rights.

- Users may download and print one copy of any publication from the public portal for the purpose of private study or research.

- You may not further distribute the material or use it for any profit-making activity or commercial gain

- You may freely distribute the URL identifying the publication in the public portal 


\title{
Perspectives of Long-Haul WDM Transmission Systems Based on Phase-Insensitive Fiber-Optic Parametric Amplifiers
}

\author{
M. Jazayerifar ${ }^{1}$, I. Sackey ${ }^{1,2}$, R. Elschner ${ }^{2}$, F. Da Ros ${ }^{3}$, T. Richter ${ }^{2}$, C. Meuer ${ }^{1,2}$, \\ C. Peucheret ${ }^{4}$, C. Schubert ${ }^{2}$, K. Petermann ${ }^{1}$ \\ (1) Technische Universität Berlin, HFT4, Einsteinufer 25, 10587 Berlin, Germany \\ (2) Fraunhofer Institute for Telecommunications, Heinrich Hertz Institute, Einsteinufer 37, 10587 Berlin, Germany \\ (3) Department of Photonics Engineering, Technical University of Denmark, DK-2800 Kgs. Lyngby, Denmark \\ (4) FOTON Laboratory, CNRS UMR 6082, ENSSAT, University of Rennes 1, F-22305 Lannion, France
}

\begin{abstract}
The deployment of phase-insensitive fiber-optic parametric amplifiers (PI-FOPAs) as inline amplifiers in longhaul WDM transmission systems is discussed, and it is outlined how to design PI-FOPAs to be a valuable upgrade option for this application.
\end{abstract}

Keywords-nonlinear fiber optics, optical parametric amplifier; long-haul optical transmission; polarization insensitive

\section{INTRODUCTION}

Fiber-optical parametric amplifiers (FOPAs) have attracted growing attention during the last years due to their promising capabilities in applications such as low-noise phase-sensitive amplification, wavelength conversion, optical phase conjugation, and signal regeneration [1,2]. In particular, FOPAs have been shown to provide broad-band gain spectra with adjustable center frequency exceeding the gain bandwidth of conventional erbium-doped fiber amplifiers (EDFAs) [1,2] which makes them appealing for bandwidth upgrades of existing long-haul wavelength-division multiplexed (WDM) transmission systems.

While phase-sensitive FOPAs offer record-low noise figures less than $3 \mathrm{~dB}$ [2], their deployment as inline amplifiers along the transmission link is challenging and might require modifications of the conventional link architecture [2]. For this reason, we restrict the discussion here to phase-insensitive fiber-optical parametric amplifiers (PI-FOPAs), which, in principle, can be seamlessly integrated into legacy transmission systems.

In comparison to EDFAs, PI-FOPAs show similar noise figure characteristics [3]. However, since FOPAs are based on efficient four-wave mixing (FWM), provided in a highly nonlinear fiber (HNLF), they are prone to introducing additional nonlinear cross-talk in WDM systems [4] in comparison with EDFA-based WDM systems. Furthermore, the inherent polarization dependence of FWM requires the use of polarization-diversity schemes.

In this paper, we review our recent results to overcome these obstacles. In section II, we discuss the nonlinear degradations in PI-FOPAs, and it will be shown that the advantage of EDFAs over PI-FOPAs can become negligible at the cost of lower power efficiency. In section III, we discuss a possible polarization-diversity scheme and its impact on the amplifier performance at high gain levels [5-7].

\section{ULTIMATE PERFORMANCE OF PI-FOPAS}

In order to obtain a large parametric gain bandwidth, it is necessary to operate near the zero dispersion wavelength of the HNLF which results in undesirable nonlinear interactions such as cross-phase modulation (XPM), cross gain modulation (XGM) due to pump depletion, and high-order FWM among the WDM channels. A Gaussian noise model has been proposed in [8] to characterize the accumulated nonlinear distortions in a long fiber link with cascaded fiber spans and amplifiers. This model has been applied in [9] to quantify the accumulated nonlinear distortions caused by PI-FOPAs in a long fiber link. It was then used to evaluate the performance of a long-haul transmission system with the following specifications: $100 \mathrm{WDM}$ channels on a 50-GHz grid, 28-GBd 16-ary quadrature-amplitude modulation (16-QAM) per channel, $10 \times 75-\mathrm{km}$ fiber spans, 15-dB PI-FOPA gain, 4.5-dB amplifier noise figure, standard single mode transmission fibers (SSMF). The inline amplifiers could be either EDFA or PI-FOPA. In Fig. 1 the resulting symbol error rate of this system is plotted versus the amplifier output power per WDM channel.

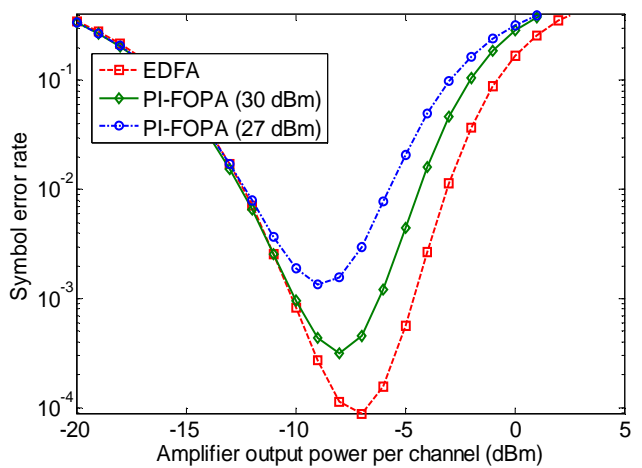

Fig. 1. Symbol error probability versus amplifier output power per channel for EDFAs and FOPAs with different pump powers $(100 \times 28 \mathrm{GBd} 16-\mathrm{QAM}$ DWDM channels, 10 spans, $50 \mathrm{GHz}$ grid)

It is observed that EDFAs and PI-FOPAs lead to the same symbol error probability at low power levels, because of the same value of noise figure. At high power levels, the EDFAbased system is limited by nonlinear crosstalk in the transmission fiber, while the PI-FOPA systems additionally suffer from nonlinear crosstalk in the HNLF. Two PI-FOPAs with the same gain were investigated. One of them had a 27$\mathrm{dBm}$ pump power (500-m HNLF) and the other had a $30-\mathrm{dBm}$ pump power (256-m HNLF). The latter outperforms the 
former because of the lower amount of FWM crosstalk among the WDM channels in the shorter HNLF. More generally, we showed that the dominant source of nonlinear crosstalk in WDM systems with small number of channels would be the nonlinear effects of the transmission fibers rather than the nonlinear impairments due to the FOPA [9]. If the number of WDM channels is large the nonlinear crosstalk due to the FOPA becomes the dominant degrading effect. The reason is that the crosstalk in the HNLF occurs among all WDM channels due to the low value of dispersion of the HNLF, while in the transmission fibers the crosstalk occurs mostly among neighboring channels due to the large dispersion value. However, from Fig. 1 it is realized that PI-FOPAs can have a performance close to EDFAs also for a WDM system with a large number of channels, but at the cost of lower power efficiency [4].

In [10] it has been experimentally shown that an OSNR penalty of less than $1 \mathrm{~dB}$ after amplification with PI-FOPA is achievable for 16-QAM signals provided that the output pump power is at least $17 \mathrm{~dB}$ larger than the overall output signal power. The experimental use of PI-FOPA as an inline amplifier for WDM systems has been demonstrated in $[2,11]$.

\section{POLARIZATION-INSENSITIVE PI-FOPAS}

The limited number of works which have been published on the experimental deployment of PI-FOPAs in long-haul transmission links is mostly due to the inherent polarization dependence of FWM, which requires the use of a dual-pump scheme with orthogonal pump polarizations [5] or a polarization diversity loop scheme [6,7] (Fig. 2)

Fig. 2 shows an experimental realization of the diversity loop scheme, in which the horizontal and vertical polarizations of the pump are transferred to the fast axis of a polarizationmaintaining (PM-) HNLF in the clockwise (CW) and counter clockwise (CCW) directions, respectively. The pump is adjusted to have the same amount of power in both directions such that both polarizations of the input signal experience the same gain in the $\mathrm{CW}$ or $\mathrm{CCW}$ directions, and the total gain becomes polarization-insensitive.

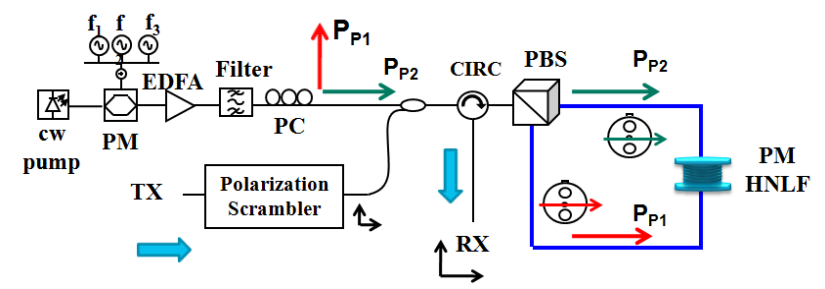

Fig. 2. Setup for the polarization-insensitive FOPA based on a diversity loop (PM: phase modulator, PC: polarization controller, CIRC: circulator, PBS: polarization beam splitter)

However, it has been shown that the Brillouin back reflections of the $\mathrm{CW}$ (or CCW) pump, may interact with the CCW (or $\mathrm{CW}$ ) pump which results in pump distortions at high pump powers, especially with pump spectra broadened by multi-tone phase modulation. This may ultimately results in distortion of the amplified signal [7]. Fig. 3 (a) shows the analytical BER evaluation of 28-GBd QPSK signals after amplification with a polarization insensitive FOPA [7]. It is observed that the BER penalty with respect to the back-to-back case increases as the gain increases. This is also verified experimentally as it can be seen in the constellation diagrams in Fig. 3 (b) [7]. Therefore, in order to have a low BER penalty, the FOPA gain should be limited. To achieve large gain with low distortion, HNLFs with low Brillouin gain coefficients should be used.

(a)
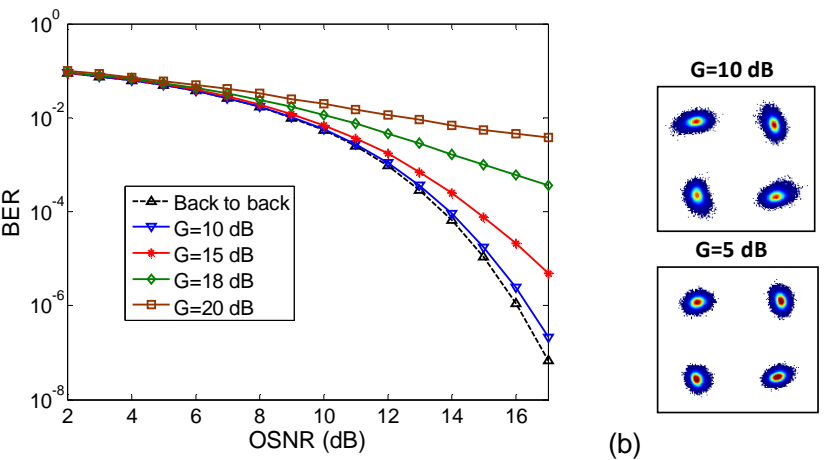

(b)

Fig. 3. (a) Analytical BER of 28 GBd SP-QPSK signals degraded by the Brillouin distorted pump (b) Experimentally measured 28-GBd SP-QPSK constellation after the polarization insensitive FOPA

\section{CONCLUSION}

Using numerical methods supported by experiments, it has been shown that the performance of PI-FOPAs in long-haul transmission links can be comparable to that of EDFAs provided that lower power efficiency is accepted. Furthermore, polarization-diversity schemes are required that allow for high signal gain and low signal distortion. For the diversity loop under investigation, it has been shown that a low Brillouin gain coefficient of the HNLF is required (together with low loss), which remains as one of the challenges for the successful deployment of PI-FOPAs as inline amplifiers in long-haul transmission.

\section{ACKNOWLEDGMENT}

This work was funded by the German Research Foundation (DFG) under projects GR 3774/1-1, GR 3774/1-2 and PE 319/26-1 and PE 319/26-2.

\section{REFERENCES}

[1] J. Hansryd et al., "Fiber-based optical parametric amplifiers and their applications," JSTQE, 8(3), pp. 506-520, 2002.

[2] M. Marhic et al., "Fiber optical parametric amplifiers for optical communication," LPR, 9(1), pp. 50-74, 2015.

[3] P. Kylemark et al., "Noise characteristics of fiber optical parametric amplifiers," JLT , 22(2), pp. 409-416, 2004.

[4] R. Elschner et al., "Characterization of FWM in duced crosstalk for WDM operation of a fiber-optical parametric amplifier," ECOC, Mo.1.A.2, 2011

[5] K. Y. Wong et al., "Polarization-independent two-pump fiber optical parametric amplifier," PTL, 14(7), pp. 911-913, 2002.

[6] I Sackey et al., "Design and performance evaluation of an OPC device using a dual-pump polarization-independent FOPA," ECOC, Tu.1.4.4, 2014.

[7] M. Jazayerifar et al., "Impact of SBS on polarization-insensitive singlepump optical parametric amplifiers based on a diversity loop scheme," ECOC, Tu.4.6.4, 2014.

[8] A. Splett et al., "Ultimate transmission capacity of amplified opticalfiber communication systems taking into account fiber nonlinearities," ECOC, MoC2.4, 1993.

[9] M. Jazayerifar et al., "Performance evaluation of DWDM communication systems with fiber optical parametric amplifiers," JLT, 13(9), pp.1454-1461, 2013.

[10] I Sackey et al., "Characterization of fiber-optical parametric amplifier in a $5 \times 28$-GBd 16-QAM DWDM system," OFC, W3E.3, 2014.

[11] Z. Lali-Dastjerdi et al., "Demonstration of cascaded in-line single-pump fiber-optical parametric amplifiers in recirculating loop transmission," ECOC, Mo.2.C.5, 2012. 